\title{
Risk management in flood events
}

\author{
G. Ciaravino ${ }^{1}$, L. Ciaravino ${ }^{1}$, G. Grimaldi ${ }^{2}$, G. Lombardi $^{2}$ \\ \& L. S. Sorvino ${ }^{2}$ \\ ${ }^{I}$ Department of Hydraulic and Environmental Engineering, \\ "G. Ippolito" University of Naples Federico II, Italy \\ ${ }^{2}$ Authority of Basin Destra Sele, Regione Campania, Italy
}

\begin{abstract}
In the present paper, areas under risk of floods are identified on the basis of the codified return periods of flood events, and appropriate river strips are then identified, with reference to which the actions of territorial planning and risk mitigation works can be defined. Hydraulic risk is determined by evaluating the expected damage to vulnerable elements (first and foremost anthropic elements) resulting from the occurrence of an event of known dangerousness. Therefore the level of risk derives from the estimate of phenomenon dangerousness (linked to the identification of the relative river strips with assigned return period) and from the vulnerability of the exposed elements. The risk is determined by means of matrixes that, even if characterized by intrinsic limitations, make it fairly straightforward to estimate a small number of parameters and hence evaluate risk level and attention level in flood phenomena for single hydrographical networks.
\end{abstract}

Keywords: flood event, hydraulic risk, mathematical model analysis, risk matrixes.

\section{Introduction}

With every passing year, the scientific community observes ongoing climate change which has, among other things, modified the values of extreme hydrological events. Demographic increase combined with rising land prices due to the shortage of building areas, has led to a disordered and indiscriminate land use with all kinds of installations being built in areas at high risk of flooding.

In conjunction with scarce monitoring and maintenance of the territory, modification of drainage networks, deforestation and topographical changes, 
these circumstances have altered both the hydraulic and the environmental equilibrium. Consequently, the increased frequency and intensity of flood phenomena, along with their rising dangerousness due to the greater vulnerability of anthropic elements, has attracted the attention of local authorities, research groups and engineers.

Hydraulic researchers are generally interested in techniques and mathematical models that are able to predict (or estimate), on the one hand, the intensity of a flood event (of given return period $\mathrm{T}$ and risk R) and, on the other, the propagation of such a flood through the hydrographical network; actually their increasingly sophisticated models may at times be somewhat complex to apply. Local authorities and technicians, on the other hand, require simple and instantly applicable tools with the following aims: identification the areas at risk; evaluation of suitable risk mitigation works; setting up of warning systems. In the latter context, in the present paper the following issues are analyzed:

- hydraulic modeling of flood phenomena with the aim of identifying floodprone areas and, therefore, river strips;

- matrixes that define levels of risk for the identified river strips.

\section{Simplified model analysis}

The identification and delimitation of hydraulic risk areas - required for the definition of mitigation works and directives to safeguard the local territory - are normally achieved through analysis of past occurrences and estimates of potential future events. Technical practice therefore tends to refer to events with a given return period $\mathrm{T}$, indicating the mean time span within which a certain event might statistically occur and be once overcome. Moreover, legislative safeguards and preventive actions aim to protect the environment by defending it from physical deterioration and reducing its vulnerability when the human sphere in particular is exposed to hydraulic risk. In general terms, the classes of elements under risk normally considered are [1]:

- built-up areas and their designated urban expansion areas;

- manufacturing areas, major hi-tech plants;

- infrastructure network and strategic lines of communication (both local and regional/national);

- important environmental areas and heritage sites;

- public and private sector utilities and services, sport and leisure facilities, hotels, etc. and primary infrastructures.

For these elements a preliminary analysis needs to be performed in order to assess the main functional characteristics as well as the potential interference (critical sections) that can arise along the various stretches of the hydraulic network when a flood event occurs.

As hydraulic transport phenomena are dependent upon the morphological processes of erosion, solid transport and subsequent deposition, in order to determine the characteristics of flood-prone river stretches, a preliminary geomorphological classification of the river network should first be conducted. This classification aims primarily to identify: 
- the river's upland (mountain) course, which cuts through strongly cohesive soils and can either destabilize the slopes at the foot of which it flows or can be subject to a concentrated solid inflow which may give rise to flood phenomena;

- the river's middle (piedmont) course, which is typically subject to deposition and may give rise to flooding if it receives considerable quantities of debris from the upstream stretches;

- the river's lowland (plain) course, located in the floodplains which are made up of soils whose morphology is such as to allow the passage of flows corresponding to return periods of $\mathrm{T}=2 \div 5$ years without the river bursting its banks, while greater flow rates (and greater values of $\mathrm{T}$ ) may give rise to flooding.

In technical practice, the mathematical computation models that are adopted for the definition of propagation phenomena and possible flooding vary in complexity according to the case in question.

In the mountain river stretches (which are often completely dry or have modest flows from springs) significantly dangerous flow rates are related to weather events which, because of the considerable slopes and the presence of a soil covering and/or water erosion of the mountain sides, can at times result in unpredictable solid transport phenomena (debris flow) [2]. In actual fact, the discontinuous and unpredictable hydraulic nature of these stretches, combined with a considerable solid transport capability, can create serious safety problems for towns or infrastructure situated in downstream areas.

For mountain stretches (which can be considered highly incised and steep) and excluding extreme solid transport phenomena such as debris flow, it can be profitably used a simplified hydraulic approach based on (i) flow characteristics in each of the critical sections, hypothesizing conditions of uniform flow or critical state, and (ii) assessments of the stream flow between bridge piers and through drains in free and submerged flow conditions. In this simplified view, hydraulic verifications can be performed using well known equations: GaucklerStrickler's equation (or equivalent) under the hypothesis of flow which is free and uniform; the equation characterizing the critical state condition:

$$
1-\frac{\alpha \cdot Q^{2}}{g \cdot A^{3}} \cdot \frac{d A}{d h}=0
$$

where $Q$ is the flow rate, $A$ is the hydraulic cross section, $h$ is the water depth, $\alpha$ is the coefficient of Coriolis, $g$ is gravity acceleration; the equation for submerged flow through an orifice which, neglecting the contractions of the fluid vein, makes it possible to calculate the head $H_{m}$ upstream from each handiwork (bridge or drain):

$$
Q=A \cdot\left(2 g \cdot H_{m}\right)^{1 / 2} .
$$

In the case of a stream flow higher than the bridge or drain, the head can be calculated by considering the flow rate $Q$ divided in two aliquot parts: the first aliquot $Q_{b}$ is the flow rate on the river bed (flow under head $H_{b}$ ) and the second one $Q_{s}$ is the flow rate over the structure (flow through weir with head $H_{s}$ ). Therefore it is possible to write the following equations: 


$$
\begin{gathered}
Q_{b}=A \cdot\left(2 g \cdot H_{b}\right)^{1 / 2} \\
Q_{s}=\mu_{s} \cdot L \cdot H_{s} \cdot\left(2 g \cdot H_{s}\right)^{1 / 2} \\
Q=Q_{b}+Q_{s} \\
H_{b}=H_{s}+B
\end{gathered}
$$

where $\mu_{s}$ is the Bélanger discharge coefficient and $L$ and $B$ are linear parameters that can be obtained from the geometry of the handiwork and the river bed. Equations (3), (4), (5) and (6) thus represent a possible system for defining the unknown quantities $Q_{b}, Q_{s}, H_{b}$ and $H_{s}$.

In the stretches which have been defined as the piedmont course (characterized by steep slope often near to critical slope), and in the plain course (generally characterized by mild slopes), it is normally worthwhile using more complex mathematical models that make it possible to evaluate the submerged flow effects caused by structures and/or morphological variations in the river bed [3]. In this case, where flooding may concern large-scale valley areas, morphological models can be employed (with comparative verification of the flood volumes) in bi-dimensional non steady flow. However, it often happens in practice that the limited topographical data render the use of such bi-dimensional models vain, as they do not provide appreciably better results than the ones that could be obtained by means of a simple (but well-applied) uni-dimensional model. In actual fact, for the definition of propagation phenomena and any flooding in longer piedmont and plain stretches (especially for fairly gentle longitudinal slopes and floods of significant duration), a uni-dimensional mathematical model can be profitably employed which, assuming boundary conditions that vary only slightly over time, is based on a succession of events of steady flow (quasi-stationary approach).

These models (usually adopted in engineering practice and of the type HECRAS and similar) are essentially based on the hydraulic equations of motion and continuity under the hypothesis of constant fluid density:

$$
\begin{aligned}
& \frac{d E}{d x}=-J \\
& \frac{d Q}{d x}=q .
\end{aligned}
$$

Equation (7) points out the principle by which the variation in specific energy $E$ (defined by Bernoulli's equation) of the stream flow per unit of distance traveled is equal to the continuous and unitary losses $J$.

The continuity equation (8) establishes the balance between masses (or, as in this case, between volumes) entering and leaving the elementary section $d x$, indicating with $q$ the lateral uniform flow. These equations are coupled with theoretical and/or experimental relations for assessing the parameters they contain as a function of the mean velocity $U$ and the water depth $h$, which are identified as the main unknown quantities in the calculation process. In particular, the relations define the assessment of the continuous load losses, the stream flow's Froude number, the transport capacity of the individual sections, and the non-uniformity of the local mean velocities (i.e.: [4-10]). 


\section{Identification of river strips}

The flow rates, taken as a basis for the hydraulic tests to identify and delimit the hydraulic risk areas, are normally determined using a statistical method based on given return periods $T$. The lack or inadequacy of field data on river flow rate values almost always leads to these flow rates being deduced from pluviometric probability curves linking the mean annual maxima for precipitation of given duration to the duration itself. The choice of the reference return periods $\mathrm{T}$ is usually related to the characteristics of the territory and the existing or planned structures in it, as well as any criteria required by law. For the above mentioned risk elements [1], it is possible to choose flow rates corresponding to values of $\mathrm{T}=5, \mathrm{~T}=30, \mathrm{~T}=100, \mathrm{~T}=300$ years.

The application of simple computation models (concisely analyzed in the preceding chapter) makes it possible to clearly identify flood-prone areas in flooding events with given return periods $\mathrm{T}$.

Thus it is possible to define:

- $\quad$ areas with a high frequency of flooding for return periods $\mathrm{T} \leq 30$ years;

- areas with a medium frequency of flooding for return periods $30<\mathrm{T} \leq 100$

years;

- $\quad$ areas with a low frequency of flooding for return periods $100<\mathrm{T} \leq 300$ years.

In these areas it is possible to distinguish between areas that are subject to direct flooding (adjacent to the river body), areas prone to flooding by upstream flows (also with transport of miscellaneous material), areas prone to flooding because of structures limiting flow (bridges with insufficient distance between piers, constrictions and/or obstructions, etc.). On this basis, we can define river strips on which to organize territorial planning and management activities [1].

A first strip, which is defined Strip 0 (S0), coincides with the ordinary flood channel, defined as the part of the water body involved in the flow of an ordinary flood corresponding to a return period $\mathrm{T}=2-5$ years. In the case of morphologically encased channels, this strip coincides with the river area located between the banks while, in the case of alluvial channels, this strip corresponds to erratic channels interested by the ordinary flood flow.

A second strip, which is defined Strip 1 (S1), corresponds to the standard flood channel which ensures the free flow of the so-called standard flood which is normally the value of the flood flow rate taken as the basis for the sizing of the hydraulic defense works: this value can be made to coincide with the flow rate corresponding to the return period $\mathrm{T}=100$ years.

A third strip, which is defined Strip 2 (S2), corresponds to flood-prone areas relative to the above defined standard flood flow. Strip 2 can be subdivided into flood-prone sub-strips with a return period $\mathrm{T}<100$ years, in which the stream flow velocity will also be taken into account as this can be a useful indicator of the intensity (and hence dangerousness) of the flood event and may at times provide a more significant estimate than the water depths. In particular, three sub-strips are identified: Sub-strip 2a (S2a) lies between the standard flood channel and the more external of the lines of water depth $h=0.30 \mathrm{~m}$ of floods 
with return period $\mathrm{T}=30$ years and of water depth $\mathrm{h}=0.90 \mathrm{~m}$ of floods with return period T=100 years; Sub-strip 2b (S2b) lies between the limit of Sub-strip 2a and the limit of the water depth $\mathrm{h}=0.30 \mathrm{~m}$ of floods with return period $\mathrm{T}=100$ years; Sub-strip 2c (S2c) lies between the limit of Sub-strip 2b and the limit of floods with return period $\mathrm{T}=100$ years.

A fourth and final strip, which is defined Strip 3 (S3), corresponds to the flood-prone areas at risk from exceptional flood events, such as the one with return period $\mathrm{T}=300$ years or by historically verified floods characterized by flow rate values well in excess of the standard flood.

In actual fact, the stream flow velocity and the location of any constrictions and/or obstructions are of great importance for flood events because of the consequences that they can produce: in particular, velocity is greater (and close to that of the central channel) in the Strips closest to the channel body (S0, S1 and S2a), while it is smaller in the more external Strips (S2b, S2c and S3).

Therefore any obstruction will exert a greater or lesser effect not only according to its shape, size and position with respect to the direction of stream flow but also according to its position with respect to stream flow in each river strip defined above.

\section{Risk matrixes}

Risk normally expresses the value of the expected damage to vulnerable elements present in the considered area, following the occurrence of an event of given dangerousness. If there are no vulnerable elements in the area, the damage and therefore the risk can be considered as null.

In other words, the level of risk is defined by the characteristics of the vulnerable element and the degree of dangerousness, i.e. the probability of an event occurring. On this basis, we can obtain an estimate of the expected damage and the risk by referring to particular conventional matrixes.

The flood-prone areas and the relative degree of dangerousness are identified by delimiting the previously defined river strips. In particular, in the case of hydraulic risk, the expression of dangerousness in area terms is provided by the flood strips, which represent the limit that flooding could reach for a given flood event; the dangerousness value is determined using the return period T.

The vulnerability of elements at risk depends on their capability to sustain the stresses caused by the event and on the intensity of the event itself (water depth, stream flow velocity, dynamics of the event). Therefore, identifying flood-prone areas as defined by homogeneous categories indicating the presence of elements of value - such as residential areas (assessed according to inhabitant number), buildings (assessed according to number and type), public buildings where users are constantly present, road and rail infrastructure - makes it possible to delimit areas at hydraulic risk. In other words, overlapping between areas of various anthropic classes (Anthropic Element Chart) and flood-prone strips (River Strip Chart) defines the areas at different risk levels (Risk Chart).

The risk $\mathrm{R}$ is normally classified using a scale of values that estimates the expected damage to the environment and anthropic elements, focusing in 
particular on the direct involvement of people. Four risk levels $\mathrm{R}$ can be defined in this way [1]:

- R1 low risk: only marginal damage to the community, the environment and the economy;

- R2 medium risk: modest damage to buildings, infrastructure and environment is possible but people's safety is not jeopardized, buildings remain operational and economic activity is not interrupted;

- R3 high risk: people's safety in jeopardy, functional damage sustained by buildings and infrastructure making them unserviceable, socio-economic activity is interrupted and the environment suffers major damage;

- R4 very high risk: possible loss of human life or serious physical injury, major damage to buildings, infrastructure and environment, destruction of socio-economic activities.

Making reference to the standard flood (taken as the basis for the sizing of the hydraulic defense works), if it is associated every type of element under risk (or area containing elements under risk) with a potential damage index $\mathrm{D}$, by means of the overlapping with the above defined river strips it is possible to identify the different levels of risk as indicated in Table 1, where D1 indicates low potential damage, D2 medium potential damage, D3 high potential damage, and D4 very high potential damage.

Table 1: $\quad$ Risk matrix.

\begin{tabular}{|c|c|c|c|c|}
\hline & S1 & S2a & S2b & S2c \\
\hline D4 & R4 & R3 & R2 & R1 \\
\hline D3 & R3 & R2 & R1 & $\mathrm{R}=0$ \\
\hline D2 & R2 & R1 & $\mathrm{R}=0$ & $\mathrm{R}=0$ \\
\hline D1 & R1 & $\mathrm{R}=0$ & $\mathrm{R}=0$ & $\mathrm{R}=0$ \\
\hline
\end{tabular}

Under the same hypotheses, Table 2 reports the index of potential damage associated to the various classes of the Anthropic Element Chart and the levels of risk derived from its overlapping with the river strips.

The obtained matrixes constitute a simple tool of synthesis, which, by identifying areas and levels of risk and damage, can help to:

- highlight the presence of critical sections, such as bridges that risk being submerged; natural or man-made constrictions, insufficient river dams, floodprone communication infrastructure;

- plan structural and non structural interventions to safeguard the local territory, such as land reclamation in hydrographic basins (by means of hydro-geological interventions as well as in areas of forest, woodland, pasture and farmland); setting and regulation of rivers; flood management; regulation of extraction activities; hydraulic maintenance, administrative regulation; monitoring and warning systems; creation of emergency plans;

- promote ecological interconnection of natural areas for the maintenance and/or gradual recovery of the river area's complexity and biodiversity. 
Table 2: $\quad$ Risk and damage associated to anthropic elements.

\begin{tabular}{|l|c|c|c|c|c|}
\hline \multicolumn{1}{|c|}{ Anthropic Elements } & Damage & S1 & S2a & S2b & S2c \\
\hline Historic City Center & D4 & R4 & R3 & R2 & R1 \\
\hline $\begin{array}{l}\text { Completion and Expansion } \\
\text { Area }\end{array}$ & D4 & R4 & R3 & R2 & R1 \\
\hline $\begin{array}{l}\text { Industrial and/or Commercial } \\
\text { Area }\end{array}$ & D4 & R4 & R3 & R2 & R1 \\
\hline $\begin{array}{l}\text { Area of community interest } \\
\text { Tourist - Accommodation } \\
\text { area }\end{array}$ & D4 & R4 & R3 & R2 & R1 \\
\hline $\begin{array}{l}\text { National \& Regional Parks } \\
\text { and Protected Areas }\end{array}$ & D3 & R3 & R2 & R1 & R=0 \\
\hline $\begin{array}{l}\text { Protected Environment Areas } \\
\text { Transport Infrastructure }\end{array}$ & D4 & R4 & R3 & R2 & R1 \\
\hline $\begin{array}{l}\text { Unauthorized constructions } \\
\text { and Isolated Houses }\end{array}$ & D4 & R4 & R3 & R2 & R1 \\
\hline Heritage Sites & D4 & R4 & R3 & R2 & R1 \\
\hline Cemeteries & D3 & R3 & R2 & R1 & R=0 \\
\hline
\end{tabular}

\section{Conclusions}

Territorial planning aims to create an organic knowledge framework of the natural and anthropic phenomena that have to be controlled by harmoniously regulating present and future land use. This planning procedure needs to be managed by means of measures that will safeguard areas at risk of flooding, preferably defined on the basis of straightforward and easily applicable procedures that can therefore be used even by technical staff with no specific expertise in this field (such as those who typically work in small administrative authorities). In this context, the simple methodology illustrated in this paper for identifying matrixes that can characterize the risk level of flood-prone areas appears to be of particular utility. These matrixes are constructed using a limited number of parameters managed by simplified models. The matrixes express the risk through the value of the damage that vulnerable elements (with hierarchic priority for anthropic elements) are expected to suffer following the occurrence of an event of given dangerousness. The identification of the flood risk stems from an analysis of the hydraulic behavior of the various sections of the river (mountain, piedmont and plain stretches) so as to identify river strips characterizing areas that are endowed with larger or smaller degree of flood dangerousness (for the various return periods T). On this knowledge it is worthwhile to base the calculation of the stream flow induced stresses and, 
therefore, the choice and entity of the interventions to be undertaken on the river, both traditional and naturalistic, which are notoriously variable over time as far as efficiency is concerned. In the final analysis, therefore, territorial planning and management activities can be facilitated by the introduction of the simple matrixes analyzed in the present paper, whose essential methodology links the hydraulic risk (and spatial danger) to the flood strips (representing the maximum floodable area in a given flood event).

\section{References}

[1] Authority of Basin Destra Sele, Piano stralcio per l'assetto idrogeologico, Final Report, Regione Campania, Italy, 2002.

[2] Ciaravino, G., Ciaravino, L., Grimaldi, G., Lombardi, G., Maddaloni, N., Minotta, C. \& Sorvino, L.S., Matrici per la valutazione dei livelli di rischio in debris flow, Giornale di Geologia Applicata, vol. 2, pp. 93-98, ISSN: 1826-1256, 2005.

[3] US Department of Transportation - Federal Highway Administration, FEWSWMS-2HD - Finite Element Surface Water Modelling System: Two-Dimensional Flow in a Horizontal Plane, User Manual, Research, Development and Technology Turner-Fairbank Highway Research Center, Publication No. FHWA-RD-88-177, 1989.

[4] Yen, B.C., Open-Channel Flow Equations Revisited, Journal of the Eng. Mech. Division ASCE, October, pp. 610-621, 1973.

[5] Reed, J.R. \& Wolkfill, A.J., Evaluation of friction slopes, River 76 Symposium on Inland Waterways for Navigation, Flood Control and Water Diversions, Colorado State University, Colorado, 1976.

[6] Blalock, M.E. \& Sturm, T.W., Minimum specific energy in compound open channel, Journal of Hydraulic Division ASCE, vol. 107(6), No. HY6, June, pp. 699-717, 1981.

[7] Feldman, A.D., HEC Models for water resources system simulation: theory and experience, Advances in Hydroscienc, vol. 12, pp. 297-423, 1981.

[8] Konemann, N., Discussion on Minimum specific energy in compound open channel by Blalock, M.E. \& Sturm, T.W., Journal of Hydraulic Division ASCE, vol. 108(3), No. HY3, March, pp. 452-464, 1982.

[9] French, R.H., Open-Channel Hydraulics, McGraw-Hill Book Company, 1985.

[10] Molinas, A. \& Yang, C.T., Generalized water surface profiles computations, Journal of Hydraulic Engineering ASCE, vol. 111(3), March, 1985. 